\title{
Model for band-edge electroluminescence from metal-oxide-semiconductor silicon tunneling diodes
}

\author{
Miin-Jang Chen, Eih-Zhe Liang, Shu-Wei Chang, and Ching-Fuh Lin ${ }^{\text {a) }}$ \\ Department of Electrical Engineering, and Graduate Institute of Electro-Optical Engineering, \\ National Taiwan University, Taipei 106, Taiwan, Republic of China
}

(Received 6 November 2000; accepted for publication 30 April 2001)

\begin{abstract}
A detailed model is proposed to explain the electroluminescence spectrum from metal-oxidesilicon tunneling diodes. This model includes phonon-assisted processes and exciton involvement. According to this model, the main peak and the low-energy tail of the electroluminescence spectrum are attributed to the transverse optical phonon and the two-phonon assisted recombination, respectively. With very few fitting parameters, the model accurately predicts the measured electroluminescence spectra. (C) 2001 American Institute of Physics. [DOI: 10.1063/1.1381000]
\end{abstract}

\section{INTRODUCTION}

Electroluminescence (EL) from Si is an important issue for monolithic integration of optoelectronics in Si ultralarge scale integration (ULSI) circuits. To improve the poor luminescent properties of $\mathrm{Si}$, many techniques have been researched. They include $\mathrm{Si} / \mathrm{SiGe}$ band structure engineering, nanocrystalline $\mathrm{Si}$, porous silicon, Er-doped $\mathrm{Si}$, and so on. ${ }^{1}$ Unfortunately, the fabrication processes for these techniques are not compatible with the standard ULSI technology. Recently, we have observed room-temperature EL from metaloxide-semiconductor (MOS) silicon tunneling diodes. ${ }^{2,3}$ The MOS tunneling diodes have the same structure as those used in ULSI circuits. In addition, the fabrication procedures are fully compatible with the present ULSI processes, showing promise for practical silicon-based optoelectronics integrated circuits. In this work, a detailed model for EL from MOS silicon tunneling diodes is presented. Physical processes involving phonon assistance and exciton-involved radiative recombination are used to fit the EL spectra. The model accurately fits the measured EL spectra with only two fitting parameters.

Because of the indirect-band gap nature of crystalline $\mathrm{Si}$, a phonon is required to provide the additional momentum for radiative recombination. At low temperature, the observed photoluminescence (PL) spectra from crystalline $\mathrm{Si}$ are attributed to exciton-involved recombination with the participation of phonons. ${ }^{4,5}$ With PL measurement, the temperature dependence of Si band gap had also been analyzed using exciton-involved radiative transitions for the temperature range up to $1000 \mathrm{~K}^{6}{ }^{6}$ MacFarlance et al. measured the absorption spectra of crystalline $\mathrm{Si}$ with high optical resolution, ${ }^{7}$ showing that the exciton process appeared in the absorption spectra of crystalline $\mathrm{Si}$ even at room temperature. These studies indicate that the optical properties of crystalline $\mathrm{Si}$ are strongly influenced by both excitons and phonons. Before recombination, an electron and a hole are first bounded to each other under the Coulomb attraction to

a) Author to whom correspondence should be addressed; electronic mail: cflin@cc.ee.ntu.edu.tw form an exciton and then recombine to emit a photon and a phonon. ${ }^{8}$ Other experimental evidence also shows that a significant number of excitons are present in $\mathrm{Si}$ at room temperature. ${ }^{9-11}$ Hangleiter deduced a strong influence of the exciton-mediated process on both the impurity and the bandto-band Auger recombination. ${ }^{9,10}$ Therefore, both excitons and phonons are taken into account in our detailed study of the EL from MOS silicon tunneling diodes.

\section{DEVICE FABRICATION AND CHARACTERIZATION}

The MOS tunneling diode in this study has the ultrathin gate oxide that allows significant current to tunnel through. The ultrathin gate oxide with thickness of $30 \AA$ was grown by rapid thermal oxidation at $900{ }^{\circ} \mathrm{C}$ on the $n$-type $\mathrm{Si}(100)$ substrate. The gas flows are $500 \mathrm{sccm}$ nitrogen and $500 \mathrm{sccm}$ oxygen at a reduced pressure. The thickness of the oxide is measured by ellipsometry and confirmed by high-frequency capacitance-voltage measurements. Transparent indiumtin-oxide (ITO) was deposited as the gate electrode by sputtering with the sample held at $150^{\circ} \mathrm{C}$. The electrode with area $1.8 \times 10^{-2} \mathrm{~cm}^{2}$ was defined by photolithography. The thickness of the Si substrate is about $500 \mu \mathrm{m}$. Aluminum was deposited on the backside as another electrode of the MOS diode. A typical current-voltage $(I-V)$ curve is shown in Fig. 1. At the voltages 4 and $-4 \mathrm{~V}$, the magnitudes of the tunneling current density are 4.3 and $2 \times 10^{-4} \mathrm{~A} / \mathrm{cm}^{2}$, respectively, showing quite fair rectification characteristics. The emission spectra correspond to the Si band gap energy pretty well. The spectra could be explained by the following theoretical model.

\section{THEORETICAL MODEL}

Based on the analysis of absorption spectra with the involvement of excitons, ${ }^{12}$ the electron and the hole must meet at the same position before the phonon-assisted radiative recombination occurs. The probability of finding an electron and a hole at the same space point can be obtained by solving the hydrogen-like Schrodinger equation of the electronhole pair, as given in the following: ${ }^{13}$ 


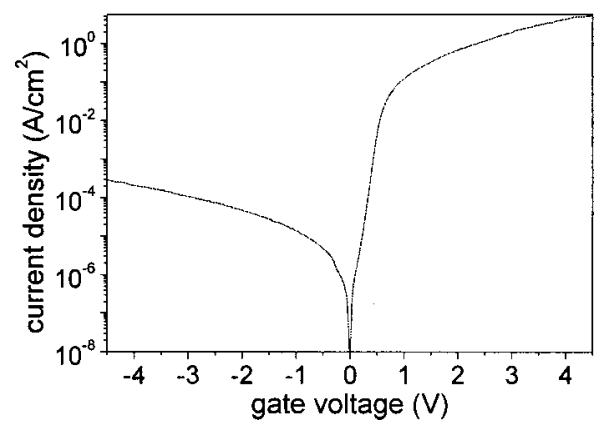

FIG. 1. $I-V$ characteristics of the $\mathrm{ITO} / \mathrm{SiO}_{2} / n$-Si tunneling diode.

$$
\begin{aligned}
& \left|\phi_{n}(\mathbf{r}=0)\right|^{2}=\frac{1}{\pi a_{B}^{3} n^{3}}, \\
& |\phi(\mathbf{r}=0)|^{2}=\frac{z \exp (z)}{\sinh (z)},
\end{aligned}
$$

where $a_{B}$ is the exciton Bohr radius; $n$ is the quantum number of the discrete exciton state; $z=\pi \sqrt{E_{B} /\left(\hbar \omega-E_{g}\right)} ; E_{g}$ is the band gap energy of $\mathrm{Si}$; and $E_{B}$ is the exciton binding energy. Equations (1a) and (1b) specify the probability of the electron and the hole at a relative separation $\mathbf{r}=0$ for the discrete and the continuum exciton states, respectively. The energies of the discrete and the continuum excitons are ${ }^{12,13}$

$$
\begin{aligned}
& E_{n}=E_{g}-\frac{E_{B}}{n^{2}}+\frac{\hbar^{2} K^{2}}{2 M}, \\
& E_{\text {cont. }}=E_{g}+\frac{\hbar^{3} k^{2}}{2 \mu}+\frac{\hbar^{2} K^{2}}{2 M},
\end{aligned}
$$

where $K$ is the center-of-mass wave vector and $k$ is the relative wave vector of exciton; and $M$ and $\mu$ are the total mass and the reduced mass of the electron-hole pair, respectively. The energy $E_{n}$ is quantized for the discrete states. The wave vectors $K$ and $k$ are related to the wave vectors $k_{e}$ and $k_{h}$ of the electron and the hole composing the exciton by the following formula: ${ }^{13}$

$$
\begin{aligned}
& \mathbf{K}=\mathbf{k}_{e}+\mathbf{k}_{h}, \\
& \mathbf{k}=\frac{m_{h}^{*} \mathbf{k}_{e}-m_{e} \mathbf{k}_{h}}{m_{e}^{*}+m_{h}^{*}},
\end{aligned}
$$

where $m_{e}^{*}$ and $m_{h}^{*}$ are the effective masses of the electron and the hole, respectively.

In the derivation of the emission spectrum due to the discrete exciton states, the expression should sum over the contributions from all the quantized exciton states. The wave vectors of the exciton and the phonon participating in the radiative recombination also need to be integrated to describe the total spectral distribution. However, the relation of the wave vectors between the electron, the hole, and the phonon must satisfy the requirement of momentum conservation. Because the exciton consists of two Fermions, the electron and the hole, such a system of two spin-1/2 particles has one singlet spin- 0 state and three triplet spin- 1 states. ${ }^{14}$ Only the singlet spin state is taken into account for luminescence due to the requirement of the symmetric wave function in the spatial coordinate $\left(\left|\phi_{n}(\mathbf{r}=0)\right|^{2} \neq 0\right.$ and $\left.|\phi(\mathbf{r}=0)|^{2} \neq 0\right)$.

Also, the spectral distribution of radiative transition is broadened as a result of the scattering of exciton by impurities, electrons, holes or phonons, etc. The Lorentzian linewidth broadening function is used to account for the scattering effect. ${ }^{15}$

$$
L\left(E_{21}-\hbar \omega\right)=\frac{1}{\pi} \frac{\hbar / \tau_{s}}{\left(\hbar / \tau_{s}\right)^{2}+\left(E_{21}-\hbar \omega\right)^{2}},
$$

where $\tau_{S}$ is the exciton relaxation time. In addition, because the total spin of the exciton is an integer, the excitons follow the Bose-Einstein statistics. ${ }^{13}$ Therefore, the emission spectrum from the discrete exciton states is given by

$$
\begin{gathered}
s(\hbar \omega) \sim \sum_{n} \frac{1}{(2 \pi)^{3}} \int_{K} \int_{q} d^{3} K d^{3} \mathbf{q} \cdot\left|\phi_{n}(\mathbf{r}=0)\right|^{2} L\left(E_{g}-\frac{E_{B}}{n^{2}}\right. \\
\left.+\frac{\hbar^{2} K^{2}}{2 M}-E_{p}-\hbar \omega\right) \cdot \delta\left(\mathbf{q}-\mathbf{k}_{e}-\mathbf{k}_{h}\right) \\
\cdot \frac{1}{\exp \left(\frac{E_{g}-\frac{E_{B}}{n^{2}}+\frac{\hbar^{2} K^{2}}{2 M}-F_{x}}{k_{B} T}\right)-1},
\end{gathered}
$$

where $E_{p}$ is the energy of the phonon used to compensate for the momentum mismatch between the electron and the hole in $\mathrm{Si}, F_{x}$ is the quasichemical potential of the exciton, $k_{B}$ is the Boltzmann constant, and $T$ is the lattice temperature. The factor $(2 \pi)^{3}$ is the volume of each quantum state in the reciprocal space. The delta function in the integral represents the conservation of momentum for the radiative recombination. Because the phonon used to compensate for the mismatch of momentum lies near the Brillouin zone edge, it is a good approximation to assume that the phonon energy $E_{p}$ is independent of the wave vector $q$. The quasichemical potential $F_{x}$ in the Bose-Einstein distribution function is determined by the total number of excitons. Assuming that the quasichemical potential $F_{x}$ of excitons is below the band gap energy $E_{g}$ over many $k_{B} T$, the Bose-Einstein function in expression (5) is reduced to the Boltzmann distribution

$$
\begin{aligned}
& \frac{1}{\exp \left(\frac{E_{g}-\frac{E_{B}}{n^{2}}+\frac{\hbar^{2} K^{2}}{2 M}-F_{x}}{k_{B} T}\right)-1} \\
& \approx \exp \left(-\frac{E_{g}-\frac{E_{B}}{n^{2}}+\frac{\hbar^{2} K^{2}}{2 M}-F_{x}}{k_{B} T}\right) .
\end{aligned}
$$

The factor $F_{x}$ can then be taken out of the integral in expression (5), so the spectral shape is not influenced by the exact position of the quasichemical potential $F_{x}$.

Similarly, the luminescence from the ionization continuum exciton states is expressed as 


$$
\begin{aligned}
S(\hbar \omega) \sim & \frac{1}{(2 \pi)^{3}} \frac{1}{(2 \pi)^{3}} \int_{k} \int_{\kappa} \int_{q} d^{3} k d^{3} K d^{3} q \cdot|\phi(\mathbf{r}=0)|^{2} \\
& \times L\left(E_{g}+\frac{\hbar^{2} k^{2}}{2 \mu}+\frac{\hbar^{2} K^{2}}{2 M}-E_{p}-\hbar \omega\right) \cdot \delta\left(\mathbf{q}-\mathbf{k}_{e}-\mathbf{k}_{h}\right) \\
& \cdot \frac{1}{\exp \left(\frac{E_{g}+\frac{\hbar^{2} k^{2}}{2 \mu}+\frac{\hbar^{2} K^{2}}{2 M}-F_{x}}{k_{B} T}\right)-1}
\end{aligned}
$$

Formula (6) should integrate over the variable $k$ since the energy of the continuum exciton states is not quantized. Be- cause we integrate over two wave vectors $k$ and $K$ for the continuum exciton states, the expression is divided by two unit volume $(2 \pi)^{3}$. Similar to the discussion for the discrete exciton states, the Lorentzian linewidth broadening function, the requirement for momentum conservation, and the BoseEinstein statistics obeyed by excitons are used in expression (6). Only the singlet spin state is considered because of the nonzero overlap of electron and hole wave functions. The Boltzmann distribution is also applied to simplify the BoseEinstein statistics.

From Eqs. (5) and (6), we obtain the total emission spectrum from both the discrete and the continuum exciton states

$$
\begin{aligned}
S(\hbar \omega) \sim & \sum_{n} \frac{1}{(2 \pi)^{3}} \int_{K} \int_{q} d^{3} K d^{3} q \cdot\left|\phi_{n}(\mathbf{r}=0)\right|^{2} L\left(E_{g}-\frac{E_{B}}{n^{2}}+\frac{\hbar^{2} K^{2}}{2 M}-E_{p}-\hbar \omega\right) \cdot \delta\left(\mathbf{q}-\mathbf{k}_{e}-\mathbf{k}_{h}\right) \\
& \cdot \frac{1}{\left(\frac{E_{g}-\frac{E_{B}}{n^{2}}+\frac{\hbar^{2} K^{2}}{2 M}-F_{x}}{k_{B} T}\right)-1}+\frac{1}{(2 \pi)^{3}} \frac{1}{(2 \pi)^{3}} \iint_{k} \int_{K} d_{q}^{3} k d^{3} K d^{3} q \cdot|\phi(\mathbf{r}=0)|^{2} L\left(E_{g}+\frac{\hbar^{2} k^{2}}{2 \mu}+\frac{\hbar^{2} K^{2}}{2 M}-E_{p}\right. \\
& \exp \left(\frac{1}{\left.\log ) \cdot \delta\left(\mathbf{q}-\mathbf{k}_{e}-\mathbf{k}_{h}\right) \cdot \frac{E_{g}+\frac{\hbar^{2} k^{2}}{2 \mu}+\frac{\hbar^{2} K^{2}}{2 M}-F_{x}}{k_{B} T}\right)-1} .\right.
\end{aligned}
$$

The temperature dependence of the band gap energy $E_{g}$ can be found in Ref. 6. The widely accepted value of the exciton binding energy $E_{B}=14.7 \mathrm{meV}^{8}$ is used for the calculation. The emission of transverse optical (TO) phonon with $E_{p}$ $=57.8 \mathrm{meV}$ is known as the dominant mechanism for momentum conservation in radiative recombination. ${ }^{4-6}$

Although the analysis of PL spectra of crystalline Si in Ref. 6 is also based on the concept of exciton-involved radiative transitions, the mathematical formula there and expression (7) are different. In Ref. 6, there are five fitting parameters. In comparison, our expression (7) needs only two fitting parameters: $T$ and $\tau_{s}$. The lattice temperature $T$ influences the linewidth through the Boltzmann distribution and the peak wavelength as a result of the temperaturedependent band gap energy $E_{g}$. The exciton relaxation time $\tau_{s}$ affects the spectral width. A major difference between our model and the analysis in Ref. 6 is the relative magnitude of luminescence from the discrete and the continuum exciton states. Instead of treating the ratio of the contributions from discrete/continuum excitons as a free fitting parameter, ${ }^{6}$ we derive this ratio from the rigorous density-of-state and statistics functions.

\section{RESULTS AND DISCUSSION}

Figure 1 shows typical $I-V$ characteristics of the $\mathrm{ITO} / \mathrm{SiO}_{2} / n$-Si tunneling diodes. When the positive gate voltage is applied, the diode operates in the accumulation region. The positive gate voltage will inject holes from ITO to $\mathrm{Si}$ and attract electrons at the $\mathrm{Si} / \mathrm{SiO}_{2}$ interface to form an accumulation layer. The tunneling holes and the localized electrons in the accumulation layer form excitons, and recombine afterward to generate radiative emission, as schematically shown in Fig. 2.

Figure 3 shows the calculated TO phonon-assisted emission spectra from the discrete exciton and the continuum exciton states at $300 \mathrm{~K}$ according to expressions (5) and (6), respectively. The discrete exciton states have stronger emission than the continuum exciton states because the discrete states have more correlation between the electron and the

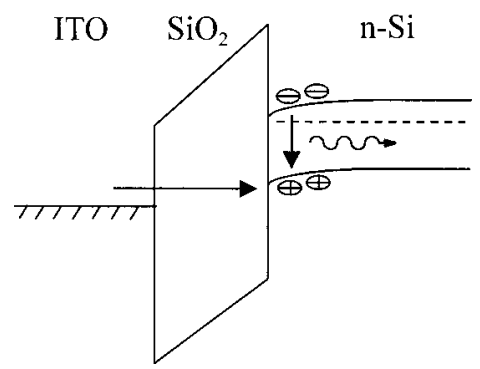

FIG. 2. Schematic band diagram of the $\mathrm{ITO} / \mathrm{SiO}_{2} / n-\mathrm{Si}$ tunneling diode operated in the accumulation region. 


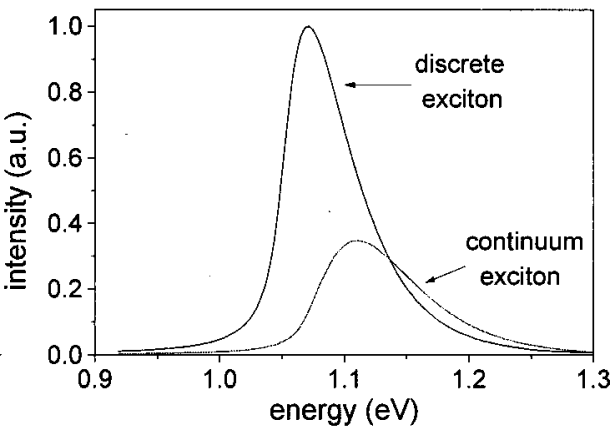

FIG. 3. Theoretically calculated TO phonon-assisted luminescence spectra from the discrete exciton states and the continuum exciton states at $300 \mathrm{~K}$, respectively.

hole. This indicates that the Coulomb attraction enhances the probability of finding an electron and a hole at the same position, hence improving the radiative recombination.

With significant current tunneling through the thin oxide, the luminescence could be observed through an infraredviewer even with a low injection current density $<1 \mathrm{~A} / \mathrm{cm}^{2}$. The diode was operated at room temperature under $\mathrm{cw}$ operation without cooling. The EL spectra of the ITO/SiO $/ 2$-Si tunneling diode are shown in Fig. 4. The diode had been operated at 3 and $3.5 \mathrm{~V}$ with injection current 88 and $133 \mathrm{~mA}$, respectively. These spectra were measured using an InGaAs detector placed at the exit of a monochromator. The dash lines are calculated spectra using Eq. (7). Temperatures at 300 and $310 \mathrm{~K}$ corresponding to the current at 88 and $133 \mathrm{~mA}$ are used for the two fitted spectra, respectively. Also $\tau_{s}=60 \mathrm{fs}$ is used for the calculation. It is in good agreement with the intraband carrier scattering time. ${ }^{16}$ With only two fitting parameters, the theoretical prediction is pretty good.

The low-energy tail in Fig. 4 is due to the two-phonon process in the radiative recombination. The PL spectrum of the MOS tunneling diode at low temperature is shown in Fig. 5. There are three major peaks corresponding to two-phonon, $\mathrm{TO}$, and transverse acoustic (TA) phonon-assisted recombination processes, respectively. ${ }^{4}$ The two-phonon process involves the emission of a momentum-conserving TO phonon and a zone center $\left(\mathrm{O}^{\Gamma}\right)$ phonon. ${ }^{4}$ When the temperature increases to room temperature, the signal of the two-phonon

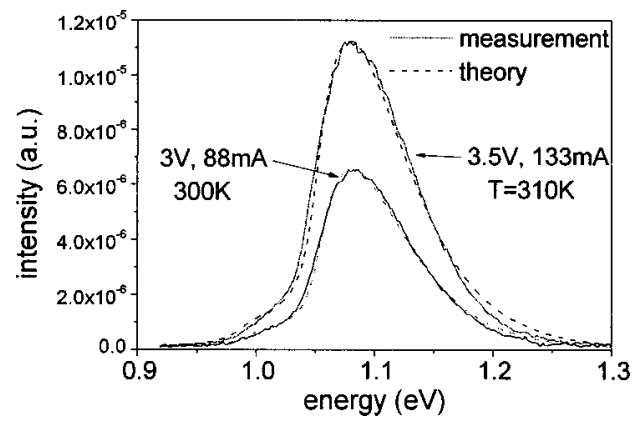

FIG. 4. Room-temperature EL spectra from $\mathrm{ITO} / \mathrm{SiO}_{2} / n$-Si tunneling diode and the theoretical fittings.

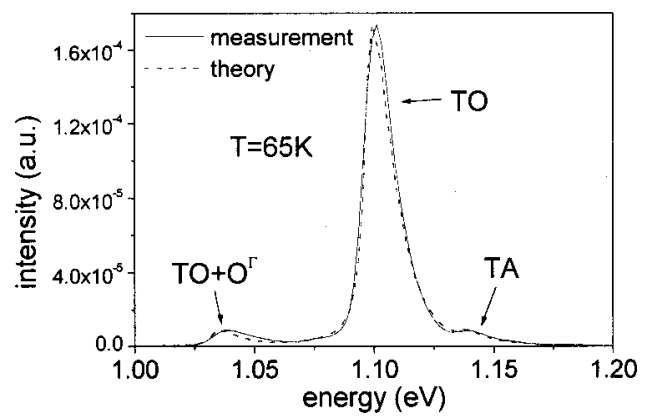

FIG. 5. Low-temperature PL spectrum from the MOS tunneling diode.

process gradually mixes with the main TO peak and forms the low-energy tail similar to Fig. 4. Decomposition of the calculated EL line shape in Fig. 4 to the main TO peak and the two-phonon signal is demonstrated in Fig. 6. The emission spectrum of the two-phonon process is obtained using Eq. (7) with $E_{p}=57.8+64.5 \mathrm{meV}\left(\mathrm{TO}+\mathrm{O}^{\Gamma}\right) .{ }^{4}$ The ratio of the magnitude between the two-phonon and the TO peaks is 0.07 , which is the same as the relative magnitude of the two signals in the PL spectrum at $26 \mathrm{~K}^{4}$ The excellent match of the theoretical spectrum to the measured data in Fig. 4 indicates that the exciton-involved radiative recombination and the participation of optical phonons take place in the roomtemperature EL from MOS silicon tunneling diodes.

We also fit the low-temperature PL spectrum in Fig. 5 using expression (7) to further confirm the validity of our theoretical model. The phonon energies $E_{p}=57.8$ $+64.5 \mathrm{meV}\left(\mathrm{TO}+\mathrm{O}^{\Gamma}\right), 57.8 \mathrm{meV}(\mathrm{TO})$, and $18.3 \mathrm{meV}(\mathrm{TA})$, are used in Eq. (7) to fit the three peaks, respectively. With the parameters $T=65 \mathrm{~K}$ and $\tau_{s}=220 \mathrm{fs}$, the linewidth and the position of peak wavelength are well predicted by the model. In the PL measurement, the cold finger is cooled at 12 $\mathrm{K}$. The temperature increase for the model could be explained by the local heating of the sample under argon laser excitation intensity $35 \mathrm{~W} / \mathrm{cm}^{2}$. Figure 5 also shows a good match between the theoretical model and the measured PL spectrum.

Possible reasons for the occurrence of EL from the MOS silicon tunneling diodes at room temperature are discussed as follows. The roughness of the $\mathrm{Si} / \mathrm{SiO}_{2}$ interface is shown to be important for EL from the MOS tunneling diodes. ${ }^{3,17}$ It has been proposed that the effect of quantum confinement

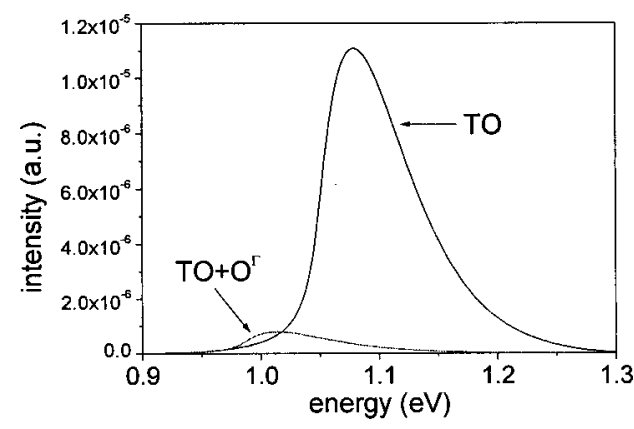

FIG. 6. Calculated emission spectra of the TO phonon and the two-phonon assisted recombination. 
will assist the formation of excitons and enhance the probability of radiative transitions. ${ }^{18,19}$ The dimension of the quantum confinement system is typically on the order of the Bohr radius of exciton. The roughness at the $\mathrm{Si} / \mathrm{SiO}_{2}$ boundary could provide the weak two-dimensional quantum confinement along the plane of the interface. The carriers are also confined in the accumulation layer due to the applied electrical field. The interface roughness and the electrical field then result in a three-dimensional quantum confinement for excitons. Therefore, in addition to the extra momentum contributed from the carrier scattering due to the interface roughness, ${ }^{3,17}$ the roughness could help in the formation of exciton for enhanced luminescence.

\section{CONCLUSION}

In summary, EL from MOS silicon tunneling diodes is analyzed using the model of phonon-assisted and excitoninvolved radiative recombination. There is an excellent fit between the model and the measured EL spectra with only two fitting parameters. The dominant peak and the lowenergy tail of the EL spectrum are attributed to the TO phonon and the two-phonon assisted recombination, respectively. The model demonstrates that the participation of optical phonons and the involvement of excitons could occur in the room-temperature EL from the MOS silicon tunneling diodes.

\section{ACKNOWLEDGMENTS}

This work is supported in part by the National Science Council, Taipei, Taiwan, R. O. C. under Contract Nos. NSC89-2215-E-002-016 and NSC89-2112-M-002-034.

${ }^{1}$ D. J. Lockwood, Light Emission in Silicon: From Physics to Devices (Academic, New York, 1998).

${ }^{2}$ C. F. Lin, C. W. Liu, M. J. Chen, M. H. Lee, and I. C. Lin, J. Appl. Phys. 97, 8793 (2000)

${ }^{3}$ C. W. Liu, M. H. Lee, M. J. Chen, I. C. Lin, and C. F. Lin, Appl. Phys. Lett. 76, 1516 (2000).

${ }^{4}$ P. J. Dean, J. R. Haynes, and W. F. Flood, Phys. Rev. 161, 711 (1967).

${ }^{5}$ J. R. Haynes, M. Lax, and W. F. Flood, J. Phys. Chem. Solids 8, 392 (1959).

${ }^{6}$ V. Alex, S. Finkbeiner, and J. Weber, J. Appl. Phys. 79, 6943 (1996).

${ }^{7}$ G. G. Macfarlane, T. P. Mclean, J. E. Quarrington, and V. Roberts, Phys. Rev. 111, 1245 (1958).

${ }^{8}$ R. A. Smith, Semiconductor, 2nd ed. (Cambridge University Press, New York, 1978).

${ }^{9}$ A. Hangleiter, Phys. Rev. Lett. 55, 2976 (1985).

${ }^{10}$ A. Hangleiter, Phys. Rev. B 35, 9149 (1987).

${ }^{11}$ D. E. Kane and R. M. Swanson, J. Appl. Phys. 73, 1193 (1993).

${ }^{12}$ T. P. McLean, in Progress in Semiconductor, edited by A. F. Gibson (Heywood, London, 1960), Vol. 5.

${ }^{13}$ H. Barry Bebb and E. W. Williams, in Semiconductor and Semimetals, edited by R. K. Willardson and A. C. Beer (Academic, New York, 1972), Vol. 8.

${ }^{14}$ E. Merzbacher, Quantum Mechanics, 3rd ed. (Wiley, New York, 1998).

${ }^{15}$ A. Yariv, Quantum Electronics, 3rd ed. (Wiley, New York, 1989).

${ }^{16} \mathrm{~K}$. Hess, Advanced Theory of Semiconductor Devices (Prentice Hall, Englewood Cliffs, NJ, 1988).

${ }^{17}$ C. W. Liu, M. H. Lee, M.-J. Chen, C.-F. Lin, and M. Y. Chern, IEEE Electron Device Lett. 21, 601 (2000).

${ }^{18}$ A. L. Efros and Al. L. Efros, Semiconductors 16, 1209 (1982).

${ }^{19}$ P. F. Trwoga, A. J. Kenyon, and C. W. Pitt, J. Appl. Phys. 83, 3789 (1998). 